\title{
A Study of Surgical Intervention in Fractures of Post-polio Residual Paralytic Lower Limb, Challenges Encountered, and Outcome Analysis
}

\author{
Sivakumar Raju ${ }^{1}$, Prahalad Kumar Singhi ${ }^{2}$, Vinoth Thangamani ${ }^{3}$, Chidambaram Muthu ${ }^{4}$
}

\begin{abstract}
Introduction: India is now a polio-free nation, but a huge load of ambulatory polio survivors present with challenging deformities and fractures to the present generation of orthopedic surgeons. These fractures are more challenging, because of the paralyzed, contracted shaft of femur fracture tissues, and inappropriate muscle forces, causing deformities. Also hypoplastic, osteoporotic, and less vascularized bone will influence the reduction maneuver, implant selection, fracture healing, and final outcome. We assess the outcome of surgical management of femoral and tibial fractures in the post-polio residual paralytic limb and the challenges encountered.

Materials and methods: Out of 22 cases with 23 fractures analyzed, 2 cases were lost to follow-up and hence excluded and the remaining 20 patients were analyzed. Pre-injury deformity, range of movement, mode of ambulation (Vignos scoring), mode of injury, surgical technique, rehabilitation, and outcomes were assessed.

Results: Minimum follow-up duration was 30 months; union was achieved by 18-24 weeks. We had one delayed union and one nonunion, which were managed with bone grafting. None of our patients had infection. A total of 16 patients regained their prefracture mobility; 4 patients developed hand to knee gait due to further weakness of the quadriceps muscle.

Conclusion: In our series, femur was the most commonly fractured bone among the post-polio residual paralytic limbs. Fractures don't heel as readily as in normal bone. Conservative treatment has guarded prognosis. Decision of surgical treatment will be challenging. Pre-injury state, surgical planning, technique, and implant choice will be key factors with appropriate rehabilitation to have a satisfactory outcome.

Keywords: Lower limb fractures, Post-polio residual paralysis, Surgical outcome, Technical challenges.

Journal of Orthopedics and Joint Surgery (2020): 10.5005/jp-journals-10079-1019
\end{abstract}

\section{INTRODUCTION}

Poliomyelitis is a viral infection caused by a neurotropic virus that causes asymmetrical flaccid paralysis due to its affection of anterior horn cells, which leads to deformities and disabilities. ${ }^{1-3}$ Though polio is nearing a complete eradication, polio survivals will still be a concern. The estimate of polio survivors is 10-20 millions of which one-third are in India. ${ }^{1,4,5}$ As these patients grow older, they are more prone for falls and sustain fractures. ${ }^{2,4}$

Abnormal muscular forces contracted paralyzed muscles and the shaft of femur fracture tissues leads to deformed joints and bones. These bones are underdeveloped, osteoporotic, and less vascular $^{6-8}$ and possess several challenges following fractures in such bones. The decision of surgical treatment in such fractures in the post-polio residual paralytic limb is critical; hasty decision leads to nasty results. Hurdles will be faced at every step in achieving reduction, appropriate implant selection, rehabilitation, and union. The role of conservative treatment should be very selective.

Paucity of literature, unique bony features, and difficulties in management of post-polio fractures has encouraged us to do this study. The aim is to assess the outcome of surgically managed fractures of femur and tibia in post-polio residual paralytic limbs and challenges encountered therein.

\section{Materials and Methods}

We prospectively analyzed fractures in lower limbs around hip and knee in post-polio limbs from 2009 to 2014 after proper approval by Institutional Review Board (IRB) of our institute. We had 22 patients
${ }^{1-4}$ Department of Orthopaedics, Preethi Hospitals Pvt. Ltd, Madurai, Tamil Nadu, India

Corresponding Author: Prahalad Kumar Singhi, Department of Orthopaedics, Preethi Hospitals Pvt. Ltd, Madurai, Tamil Nadu, India, Phone: +91 8122192632, e-mail: docpsin2001@yahoo.co.in

How to cite this article: Raju S, Singhi PK, Thangamani V, et al. A Study of Surgical intervention in Fractures of Post-polio Residual Paralytic Lower Limb, Challenges Encountered, and Outcome Analysis. J Orth Joint Surg 2020;2(1):10-16.

Source of support: Nil

Conflict of interest: None

with 23 fractures -17 fractures in femur and 6 in tibia in the paralytic limb; all were managed surgically except two patients who lost to follow-up. So, we included 20 patients with 21 fractures in our study.

Inclusion criteria-fractures of femur and tibia in post-polio paralytic limb. Fractures other than femur and tibia were excluded; patient not willing to be a part of the study were excluded. Conservatively treated patients were also excluded.

The demographic data, pre-injury Vignos score, mode of injury, fracture pattern, surgical intervention, follow-up, complications, and results were analyzed.

Number of male were 13 (65\%) and female 7 (35\%), 67\% sustained fractures following trivial fall and $33 \%$ had road traffic accident (RTA), 16 patient had fractures of femur of which 4 were involving neck, 4 had distal femur, and 8 had shaft fractures. Four patients had tibial

(c) The Author(s). 2020 Open Access This article is distributed under the terms of the Creative Commons Attribution 4.0 International License (https://creativecommons. org/licenses/by-nc/4.0/), which permits unrestricted use, distribution, and non-commercial reproduction in any medium, provided you give appropriate credit to the original author(s) and the source, provide a link to the Creative Commons license, and indicate if changes were made. The Creative Commons Public Domain Dedication waiver (http://creativecommons.org/publicdomain/zero/1.0/) applies to the data made available in this article, unless otherwise stated. 
fractures, one was type VI Schatzker, two posterior cruciate ligament (PCL) avulsions, and two were shaft fractures; all patients underwent surgical management. Minimum follow-up duration was 30 months.

Four distal femur fractures were managed with opposite side proximal tibia lateral locking compression plate (LCP) (Fig. 1) was used to accommodate the hypoplastic bone in three cases and the contoured recon plate was used in another one case. In neck of femur, two were managed with closed reduction and internal fixation (CRIF) with cancellous screws and one with dynamic hip screw (Fig. 2) and one with uncemented total hip replacement

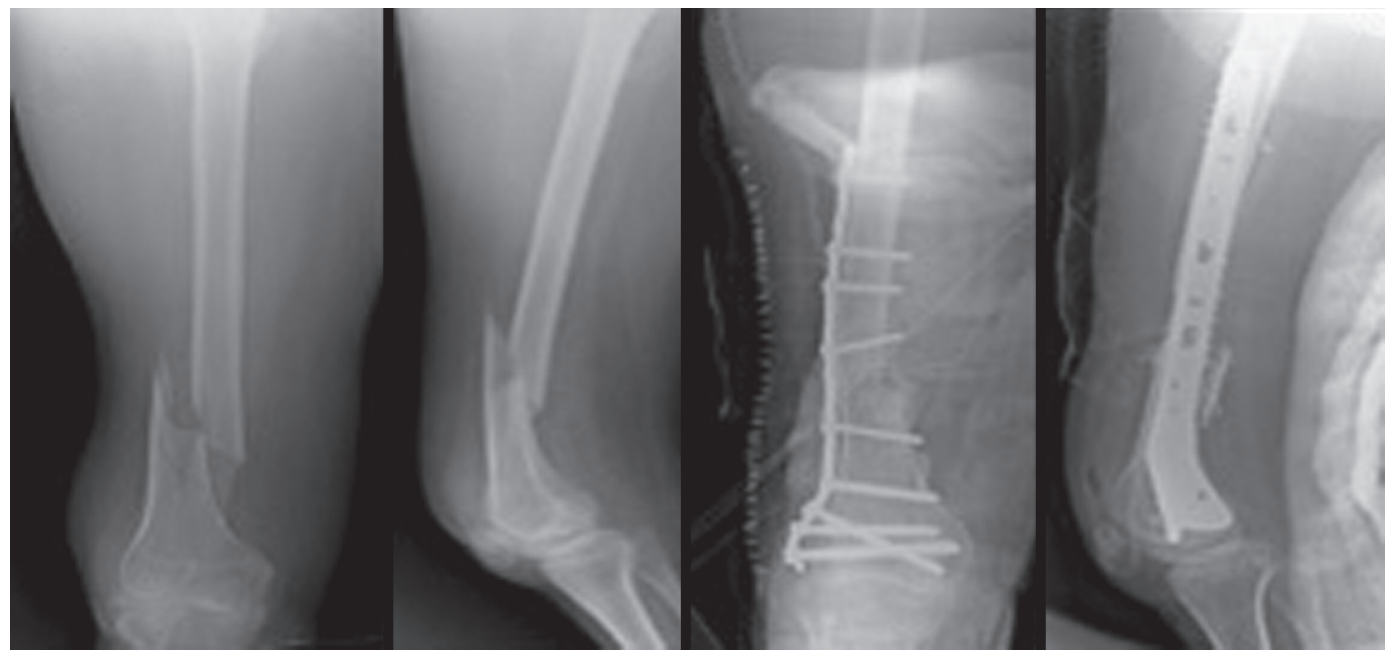

Fig. 1: Supracondylar femur fracture managed with opposite site proximal tibia lateral LCP

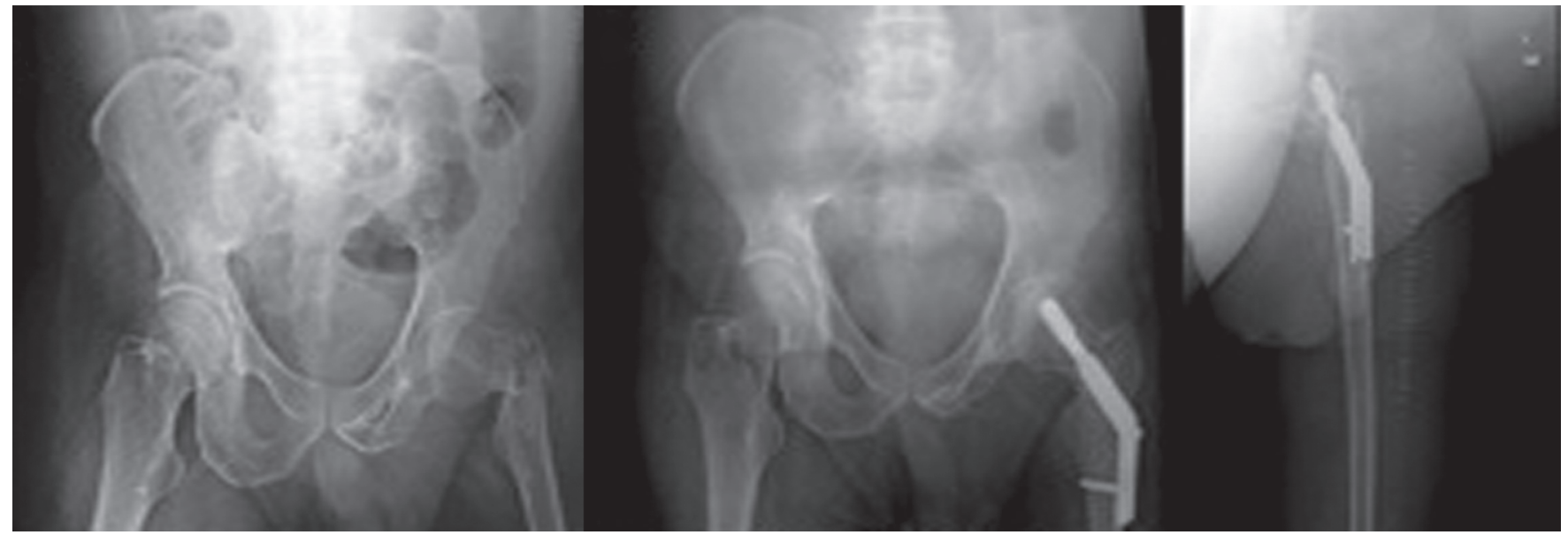

Fig. 2: Basicervical neck fracture managed with the DHS plate was needed to cut to accommodate the hypoplastic femur

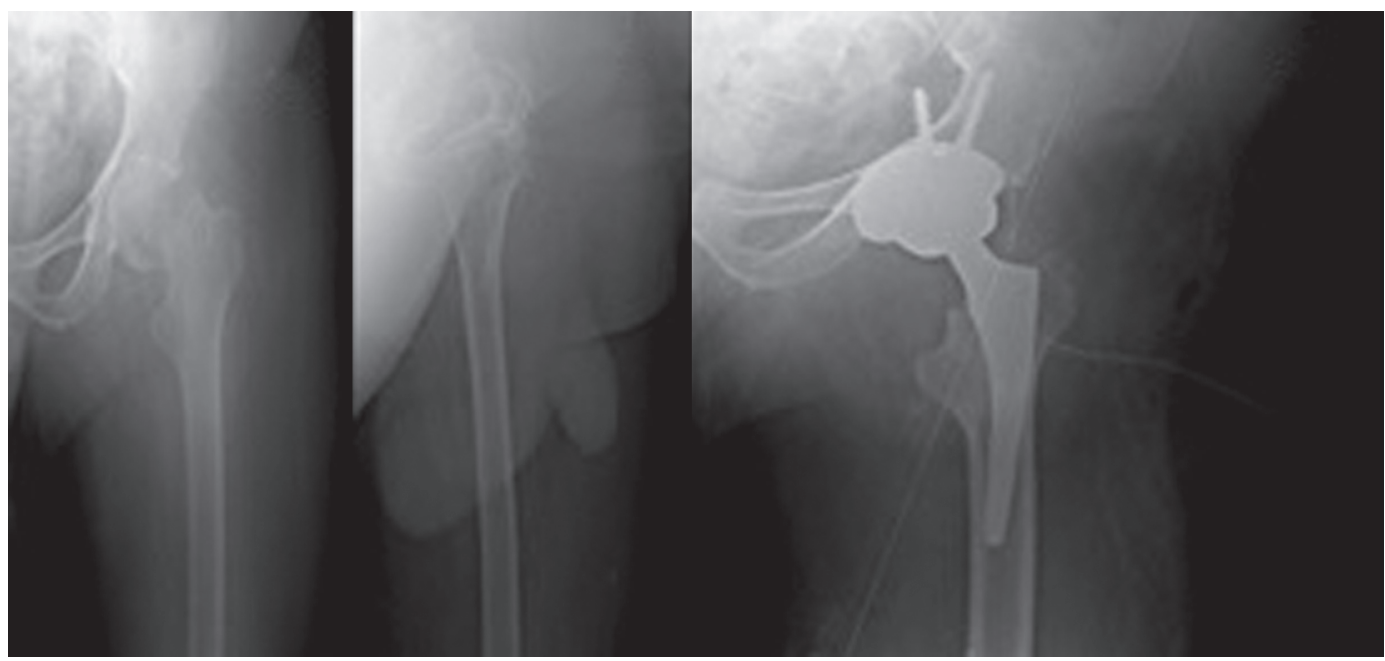

Fig. 3: Neck of femur with good muscle power managed with THR 


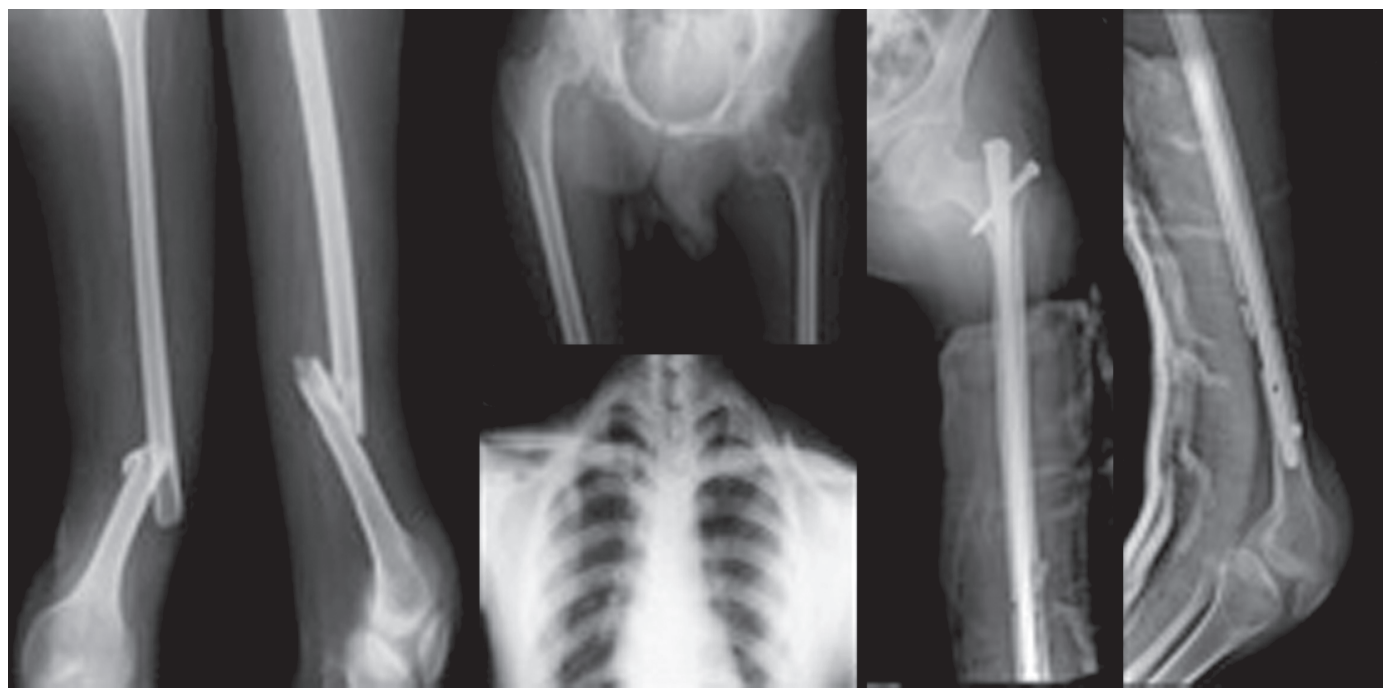

Fig. 4: Femoral shaft with deformed anatomy fracture managed with IMILN $(8 \times 280 \mathrm{~mm}$ size nail)

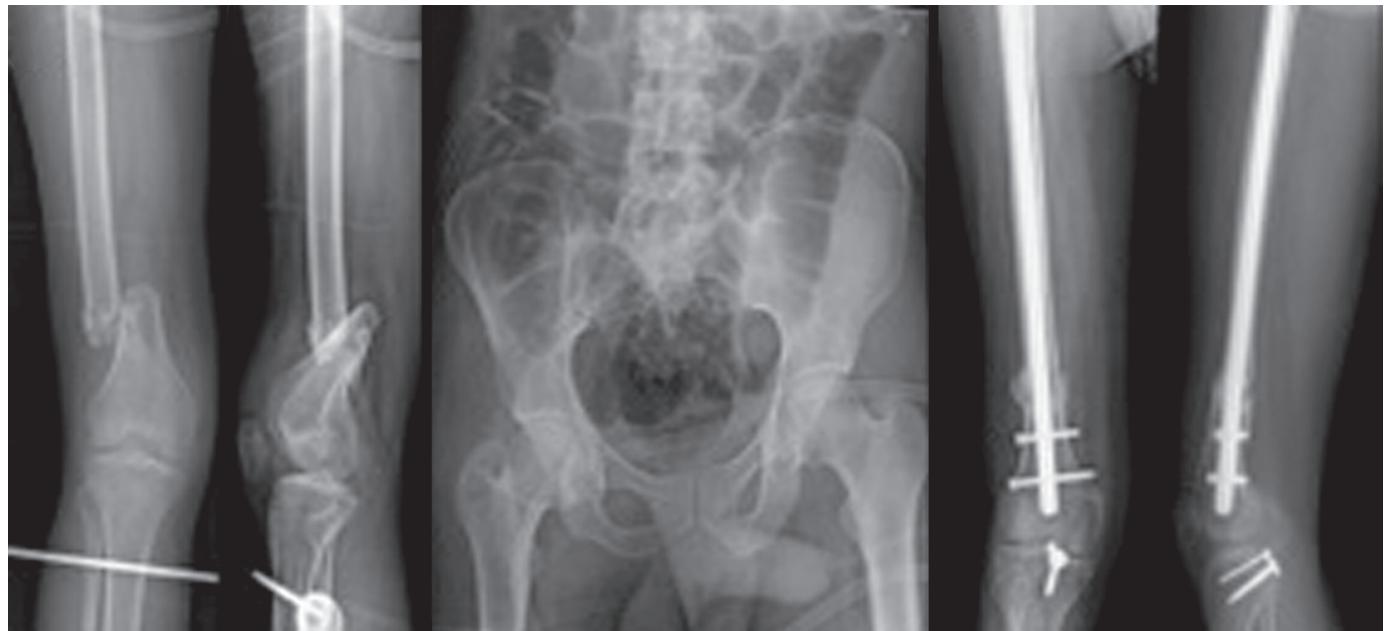

Fig. 5: Distal femoral shaft fracture with PCL avulsion pre- and postoperative

(Fig. 3) where there were not much abnormal muscular forces. In femoral shaft fractures, CRIF with titanium elastic nails was done in one and open reduction and internal fixation (ORIF) with plating in another one patient. Out of the six intramedullary interlocking nailing (IMILN) (Fig. 4), open reduction was done in two cases.

Of the two cases of PCL avulsion, fracture one was isolated and other was associated with shaft of femur fracture, where open reduced by the Burk and Schaffer's approach was done and fixed with cancellous screws (Fig. 5). Type VI Schatzker proximal tibia fracture was treated with open reduction and dual plate fixation with bone grafting (Fig. 6). Two tibial shaft fractures were managed with CRIF with intramedullary nailing. Postoperatively, patients were protected in splints and braces in the early period and were mobilized gradually.

Technical difficulties and decision making during management of these fractures were crucial. Like whether to use fracture table or not, entry point in deformed and hypoplastic proximal femur, joint contractures, and tight iliotibial band were certain factors which influenced the reduction; gentle traction in the previous deformed position enables the reduction. The narrow medullary canal and deformed bones governed the choice of implant.
Most of the shaft fractures had flexion deformities, and reduction was achieved in same position. Proper preoperative planning is very important, as commonly available sizes are not suitable; customized nails, which would suit the diameter of the canal and appropriate length, titanium elastic nails, and all varieties of plate be arranged beforehand. Master chart of our case series is shown in (Table 1).

Fracture healing was assessed radiologically at regular intervals and functionally using the Vignos scale for lower extremity.

\section{Results}

Average fracture union was achieved around 18-24 weeks; one patient had delayed union and one nonunion. On the Vignos scale, 16 patients regained the pre-injury level, but 4 patients dropped down by one level. One of our patient participated in Asian Paralympics games.

\section{Discussion}

Though polio is getting extinct and eradicated in most parts of the world, but polio survivors have grown old with sequelae of the deformed paralytic limb. ${ }^{1-3}$ Though there is asymmetric 


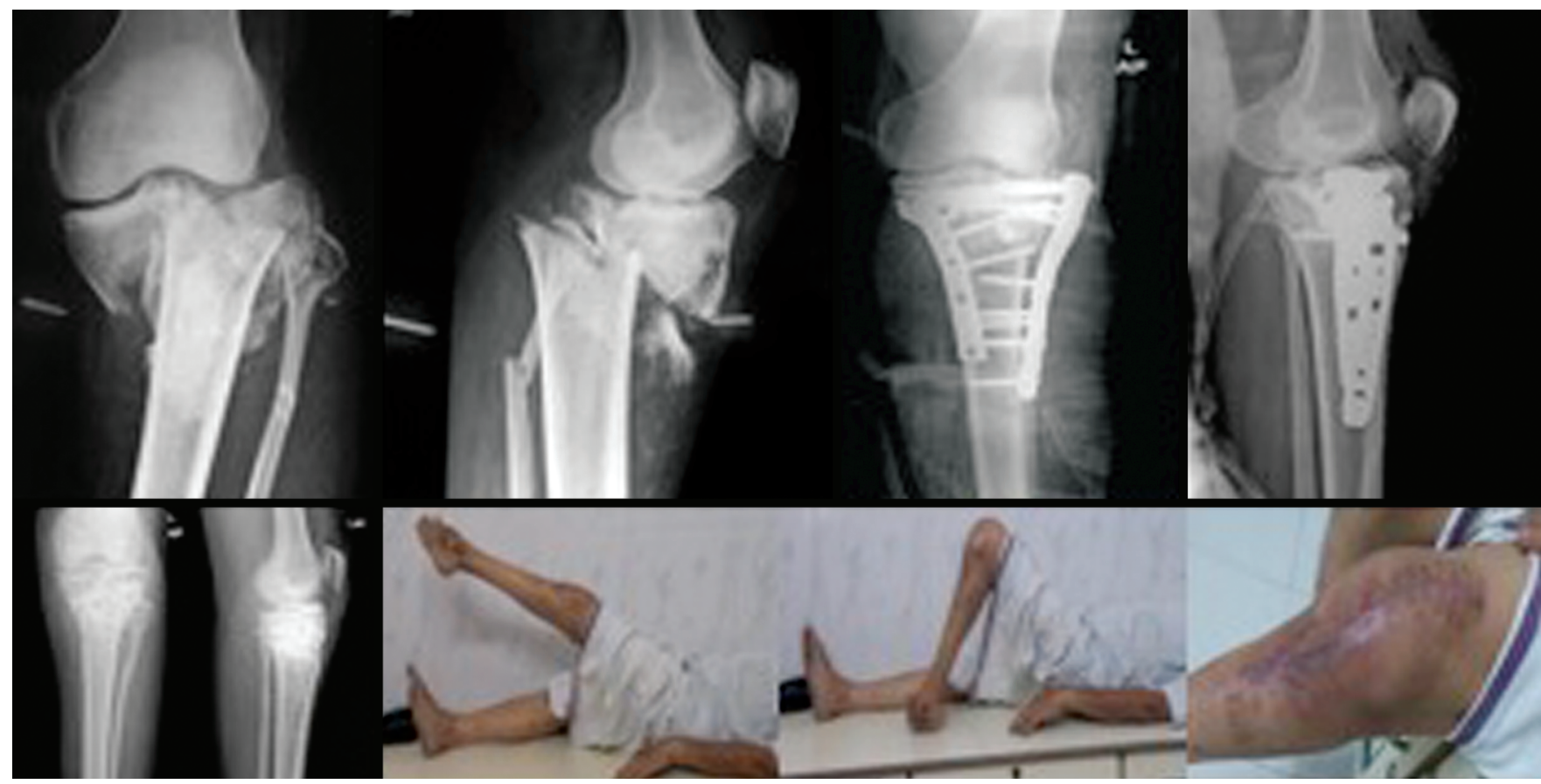

Fig. 6: Schatzker VI fracture managed with dual plating with excellent outcome

involvement, lower extremity are more commonly affected in polio and have a small deformed flail limb, more prone for fractures following low-velocity injury. A study by Goerss et al. on polio survivors showed that there is a $48 \%$ chance of any fracture after 40 years of age. They concluded that there's unusual risk of fracture in affected poliotic limbs and low-energy proximal shaft, and distal femoral fractures are more common. ${ }^{1}$ Bickerstaffe et al. have suggested that frequency of fall in these patients is four times higher than the normal population, and further more in elderly population. About $50-84 \%$ of polio patients sustain a fall during their lifetime. ${ }^{5}$ The flaccid asymmetric paralysis and muscle atrophy influences the stability, range of movement, contractures, and alters the gait pattern that predisposes to fall. There are several explanations or contributing mechanisms for frequent falls. Quadriceps weakness itself is a major risk factor for falls, as there is preponderance of lower limb involvement in polio. Knee buckling due to quadriceps weakness during the stance phase and failure to clear the foot off the ground in the swing phase due to ankle dorsiflexor or hip flexor paralysis again frequently lead to falls. ${ }^{5}$ In addition, the gait is imbalanced in these patients due to deformities, contractures, limb length discrepancies, and improper distribution of muscle forces.

Osteoporosis or osteopenia is one of the most common associations of post-polio residual paralytic limb but the exact prevalence is not known and regional osteoporosis is characteristic to this condition. ${ }^{9}$ A study by Bickerstaffe says $96 \%$ of polio patients had osteopenia and osteoporosis and reported a fracture incidence of $38 \%$ over 5 years. ${ }^{5}$ The site most commonly affected by osteoporosis in such cases is usually the hip. ${ }^{2}$ The lifetime risk of sustaining a proximal femur fracture rises with the decrease in bone mineral density (BMD). A significant correlation between bone mass and muscle strength is well documented and reduced total lean muscle mass is an important contributing risk factor. The poliotic limbs have a lower BMD than rest of the skeleton and this predisposes them to fractures following trivial or significant fall. Chang et al. found that the femoral neck BMD was lower both in normal and affected limb in polio patients as compared to age-matched controls, though the paralyzed side was more osteopenic than the normal one. Haziza et al. showed that the hip was the most common site to be involved; another study confirmed reduced $B M D$ of the femoral neck region well compared to normal population. ${ }^{2}$ Identifying the osteopenia early and administration of bisphosphonates were effective to decrease the incidence of fractures. ${ }^{9}$

It is difficult to achieve pre-injury ambulatory status in these patients after fracture. Very few studies like the one by Naude et al. are in favor of conservative treatment in the form of traction, bracing, and casting for fractures in polio patients; healing of fractures can be delayed but in their paper they concluded that healing progresses at normal rate, without any excess callus or hypertrophic callus. ${ }^{10}$ We had an antenatal patient with a precious baby who had a minimally displaced distal femur fracture that was treated conservatively; otherwise, most of fractures were surgically fixed. Long period of immobilization and nonweight bearing can contribute to osteoporosis, delayed fracture healing, and can worsen the prognosis; $;, 11$ also the risks of malunion, nonunion, joint contractures, and pressure sores are common association. ${ }^{12,13}$ This does not mean surgical management is immune of complications; despite the best surgical efforts, hardware failure and subsequent reoperation rate are higher in some cases. The primary aim of the treatment of these fractures should be achieving early mobilization, union, and rehabilitation to prevent joint contractures and further muscle wasting. Teamwork by orthopedic surgeon, orthotist, and physiotherapist will give a satisfactory functional outcome.

Factors like underdeveloped, osteoporotic, deformed hypovascular bones and shaft of femur fracture tissue contracture with deformities of joints pose several challenges in the management of these fractures. 


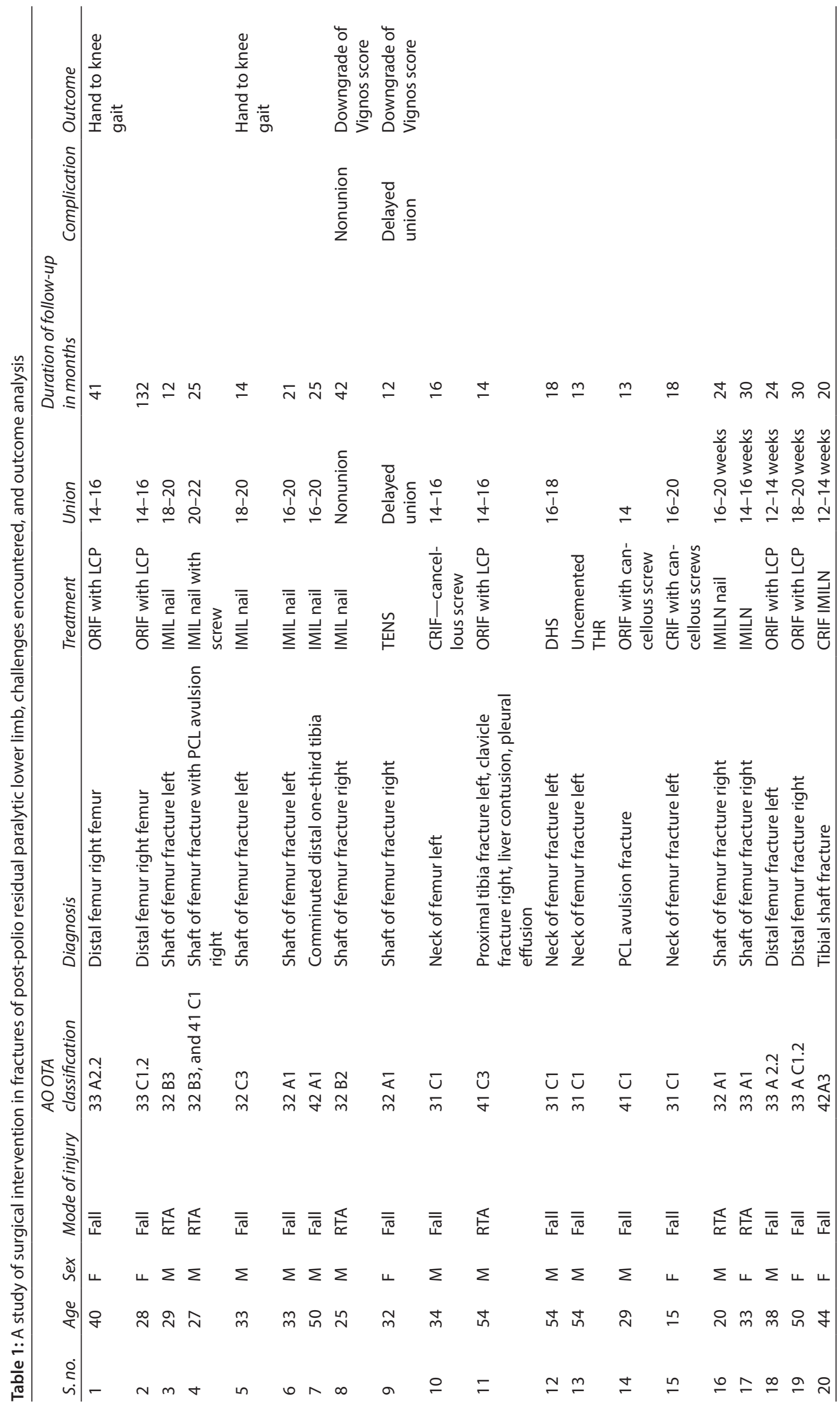


Narrow intramedullary canals, deformed small bones, and contracture of joints make insertion of standard-sized nails difficult; also access to correct entry points is hampered. With limb length discrepancy, assessing the length of the nail preoperatively is also difficult. ${ }^{13}$ In some cases, with extreme bony distortions intramedullary devices like titanium elastic nailing system or Rush pins should be considered for fixation. The elastic nail aligns the fracture fragments but rotational instability remains a concern in adult bone, so additional splinting is needed. Positioning the patient over a fracture table for nailing may be difficult. It might not be possible to use the traction piece for reduction because of flexion abduction deformity in the hip and flexion deformity in the knee, which are common in these patients. The patient can be operated in the supine or lateral position as per the surgeon's preference. $^{13}$ In cases where normal anterior bowing is absent, it is advisable to use undersized nail to negotiate it easily without causing an iatrogenic fracture; we had this in one case that was appropriately managed by proximal locking. The deformity at the fracture site is usually less compared to the non-polio patients due to lack of muscle spasticity and poor muscle bulk. But reduction can sometimes be really difficult because of the contracted iliotibial band. Hence, manual traction with different degrees of knee flexion is sufficient to align the fracture. Strong traction should be avoided in flaccid limb of polio patients as it may result in overdistraction or displacement of fracture fragments. ${ }^{13}$ The above-mentioned challenges with intramedullary nails can be overcome by the use of external fixators or plates described. One should not hesitate to do an open reduction in case of difficult reduction.

Proximal femur fractures where regional osteopenia is most commonly seen and with small, valgoid, anteverted femoral neck there is greater risk of difficult reduction, nonunion, and implants failure. ${ }^{14}$ When dynamic hip screw or cannulated cancellous screw fixation is not possible, proximal femur locking plates or rarely external fixators may be useful. Nonunion is very difficult to manage. Arthroplasty still remains an option in some cases where muscle power is reasonably good. A complete set of implants with various sizes has to be kept to avoid intraoperative hassles. In one case, we had to cut the dynamic hip screw plate to make it seat on the bone; only one purchase distally was possible.

Plates are very suitable for metaphyseal and some diaphyseal fractures where intramedullary fixation is difficult due to deformed and narrow bones. Locking compression plates have revolutionized the plate osteosynthesis particularly in the case of osteoporotic fractures where it acts like an internal fixator which provide an additional "cortex", i.e., the plate itself for screw purchase and distribute the loads equally at all plate-screw interfaces, thus increasing the pull-out strength and improving stability. Plating irregular deformed bones require plate contouring. ${ }^{13}$ Protection of the internal fixation with bracing or plaster slab for initial period reduces the risk of construct failure, periosteal stripping, loss of fracture hematoma, and blood loss. Minimally invasive techniques has minimal role in these fractures. Most advanced is the computerassisted designing (CAD) of implants that suits patient's bony geometry and use of the navigation system. ${ }^{15}$

Postoperative regime and rehabilitation are more demanding. One should avoid immobilization for longer periods; at the same time, appropriate braces or orthotic support and proper rehab protocol will give a satisfactory outcome. Obesity, social causes, motivation, or mental state of the patient also has implications on the rehabilitation and outcome. Nutritional calcium supplements and sometimes bisphosphonates do help in achieving union.

Never try to achieve the correction of preexisting deformities in the fractured limb. Knee flexion deformity, which can be corrected by a distal femoral extension osteotomy, sometimes can be achieved in cases of distal femur fracture. ${ }^{16}$ It will tempt us to do so or sometimes patient may also request for it, but this change in biomechanics may sometimes be difficult to adapt in an adult patient. Adaptability in children is remarkable and accepts biomechanical changes, but it is not the case in adults. ${ }^{17}$ So, achieving the pre-injury level remains the main task.

\section{Conclusion}

The femur is most commonly fractured bone in post-polio adults; osteoporosis with trivial fall being the most common cause of fracture. Fractures do not heel as readily as normal bone and conservative treatment has limited indications.

Decision making is the key. Surgical intervention will never be easy tasks; hurried decision has poor fate. Anticipating the surgical problem and appropriate preparation in advance to tackle this will help one to come out successful.

The operating surgeon should be prepared with complete armamentarium of implants like smaller intramedullary nails with smaller length, titanium elastic nails or rush nails, assorted-size $\mathrm{LCP}$, etc. Thorough preoperative planning is very important; one has to know preexisting deformities, shape, and quality of the bone, so it helps in achieving satisfactory reduction and fixation with appropriate implants. Postoperative rehabilitation will be demanding and it also influences the outcome.

\section{ACKNoWledgment}

Department of Orthopedics, Preethi Hospitals P Ltd. This manuscript was neither presented as part or whole at any scientific meeting nor published anywhere earlier. This manuscript has been read by all the authors approved and represents honest work.

\section{References}

1. Groce NE, Banks LM, Stein MA. Surviving polio in a post polio world. Soc Sci Med 2014;107:171-178. DOI: 10.1016/j.socscimed.2014.02.024.

2. Haziza M, Kremer R, Benedetti A, et al. Osteoporosis in a postpolio clinic population. Arch Phys Med Rehabil 2007;88(8):1030-1035. DOI: 10.1016/j.apmr.2007.05.010.

3. Emara KM, Khames A. Functional outcome after lengthening with and without deformity correction in polio patients. Int Orthop (SICOT) 2008;32(3):403-407. DOI: 10.1007/s00264-007-0322-0.

4. Bhandari N. After eradication: India's post-polio problem. BMJ 2014;348:g2275. DOI: 10.1136/bmj.g2275.

5. Bickerstaffe A, Beelen A, Nollet F. Circumstances and consequences of falls in polio survivors. J Rehabil Med 2010;42(10):908-915. DOI: 10.2340/16501977-0620.

6. Faraj AA. Poliomyelitis: Orthopedic management. Curr Orthop 2006;20:41-46. DOI: 10.1016/j.cuor.2005.10.005.

7. McComas AJ, Quartly C, Griggs RC. Early and late losses of motor units after poliomyelitis. Brain 1997;120(8):1415-1421. DOI: 10.1093/ brain/120.8.1415.

8. Lloyd ME, Spector TD, Howard R. Osteoporosis in neurological disorders. J Neurol Neurosurg Psychiatry 2000;68(5):543-547. DOI: 10.1136/jnnp.68.5.543.

9. Alvarez A, Kremer R, Weiss DR, et al. Response of postpoliomyelitis patients to bisphosphonate treatment. PMR 2010;2(12):1094-1103. DOI: 10.1016/j.pmrj.2010.08.009. 
10. Naude RJ, Spencer RF. Late effects of poliomyelitis - a review of capacity. S Afr Med J 1990;77(11):575-576.

11. Sharrard WJ. Paralytic deformities of the lower limb. Int Orthop (SICOT) 1984;8(2):147-154. DOI: 10.1007/BF00265836.

12. Vignos Jr PJ, Archibald KC. Maintenance of ambulation in childhood muscular dystrophy. J Chronic Dis 1960;12(2):273-290. DOI: 10.1016/0021-9681(60)90105-3.

13. El-Sayed Khalil A. Locked plating for femoral fractures in polio patients. Arch Orthop Trauma Surg 2010;130(10):1299-1304. DOI: 10.1007/s00402-010-1126-z.

14. Babu SS, Babu TVS, Kiran KR, et al. Difficulty in fixation of fracture neck of femur in a paralytic hip: a case report. J Evol Med Dent Sci 2014;3(30):8484-8489. DOI: 10.14260/jemds/2014/3074.
15. Gupta M, Jain VK, et al. Comprehensive review of challenges associated with management of lower limb fractures in poliomyelitis patients. J Clin Orthop Trauma 2016;7(4):276-281. DOI: 10.1016/j. jcot.2016.08.007.

16. Zimmerman $\mathrm{MH}$, Smith CF, Oppenheim WL. Supracondylar femoral extension osteotomies in the treatment of fixed flexion deformity of the knee. Clin Orthop Relat Res 1982(171):87-93. DOI: 10.1097/00003086-198211000-00015.

17. Thirunarayanan V, Ramprasath DR, Rajan A. Assessment of bone geometry and its considerations in implant selection for polio affected femoral fractures: An outcome analysis. Int J Orthod Sci 2017;3(1):441-448. DOI: 10.22271/ortho.2017.v3. i1f.65. 